\title{
LES ADN
}

$\mathbf{m} / \mathbf{S}$

médecine/sciences $1994 ; 10: 953-5$
TOPO-ISOMÉRASES, GARDE-BARRIËRES

DU GÉNOME,

ET LEUR SABOTAGE

PAR LES ANTIBIOTIQUES

ET LES ANTICANCÉREUX

\section{Yves Pommier}

\section{ADRESSE}

Y. Pommier: chief of the INA topology section. Laboratory of molecular pharmacology, Building 37, Room 5C25, National Cancer Institute, National Institutes of Health, Bethesda, MD 20892, États-Unis. 'importance fonctionnelle des ADN topo-isomérases est illustrée par leur caractère ubiquitaire puisqu'elles ont été identifiées aussi bien dans les plantes que dans les bactéries ou les cellules eucaryotes normales ou malignes. Com-me l'expliquent clairement J.F. Riou et M. Duguet dans leur article (p. 963 de ce numéro), la structure en hélice de l'ADN induit des contraintes topologiques que les topo-isomérases résolvent afin de permettre la séparation des deux brins de l'ADN lors des processus de transcription, de réplication et de réparation de l'ADN. Pour ce faire, les $\mathrm{ADN}$ topo-isomérases ouvrent et ferment les ponts phosphodiesters de l'ADN en formant des complexes enzymatiques de clivage, sorte de "barrières topologiques". Ce mode d'action les distingue des hélicases (" déroulases ") qui séparent les deux brins de l'ADN en rompant les liaisons hydrogènes entre deux bases complémentaires.

Les ADN topo-isomérases sont classées en deux groupes fonctionnels suivant qu'elles ouvrent et referment les barrières topologiques (liaisons phosphodiesters) sur un seul ou sur les deux brins de l'ADN [1-3]. I.es ADN topo-isomérases de type I (topo-isomérase I ou top l) induisent des cassures sur un seul des deux brins d'ADN tandis que les ADN topo-isomérases de type II (topo-isomérase II ou top 2) induisent des coupures double-brins. La top 2 est indispensable à la survie cellulaire, ce que démontrent les expériences faites sur des souches mutantes de levure qui meurent aux températures non permissives pour l'expression de la top 2. En l'absence de top 2, les chromosomes ne se séparent pas lors de la mitose, car les boucles d'ADN ne peuvent passer au travers les unes des autres. Au contraire, l'absence de top l est compatible avec la survie de la levure, probablement parce que la top 2 prend la relève fonctionnelle de la top 1. Cela n'est plus vrai dans les cellules de mouches drosophile [4] et de mammifères dans lesquelles la top 1 est indispensable à la survie cellulaire, vraisemblablement du fait de la plus grande complexité de leur génome comparée à celle du génome des levures.

Certains virus tels que celui de la vaccine et, peut-être, ceux respon- 
sables de l'immunodéficience humaine, codent et produisent une topo-isomérase de type I. Le virus de la vaccine a besoin de sa propre topo-isomérase pour se répliquer dans le cytoplasme de la cellule infectée dont les topo-isomérases sont concentrées dans le noyau. La partie carboxy-terminale du virus de la vaccine présente d'importantes analogies de séquence avec la partie carboxy-terminale de la top 1 eucaryote, alors qu'elle est amputée des deux tiers correspondant à la partie amino-terminale. Ainsi, son poids moléculaire n'est que de $30 \mathrm{kDa}$ alors que celui de la top 1 eucaryote est de $100 \mathrm{kDa}$. La top 1 virale est dépourvue des séquences nécessaires à une translocation nucléaire et reste dans le cytoplasme où elle participe à la réplication du virus.

L'importance fonctionnelle des topo-isomérases est encore attestée par la constatation expérimentale suivante: la surexpression de ces enzymes dans une cellule induit la mort de cette cellule. Cette mort cellulaire est probablement la conséquence de mutations de l'ADN dues à une hyperactivité de clivage et de religation de l'ADN par les topo-isomérases. Par conséquent, le niveau d'expression des topo-isomérases est étroitement lié aux besoins fonctionnels de la cellule. Il est donc étroitement contrôlé, soit au niveau génique, soit au niveau protéique (phosphorylation, poly-ADP-ribosylation, ubiquitinylation, couplage à d'autres protéines). Du fait de l'impossibilité de surexprimer les topo-isomérases dans les cellules, on ne dispose pas encore de quantités d'enzyme suffisantes pour effectuer leur cristallisation. Celle-ci serait pourtant très utile, d'abord pour analyser les mécanismes catalytiques de ces enzymes et leur inhibition par divers médicaments, puis pour rechercher de nouveaux inhibiteurs encore plus actifs et plus spécifiques. Un grand nombre d'antibiotiques et d'agents cytotoxiques agissent en bloquant les topo-isomérases, c'est-àdire en empêchant ("sabotant") l'ouverture et la fermeture des barrières topologiques (complexes de clivage). La plupart de ces produits vité bactéricide ou cytotoxique a été identifiée avant que l'on mette en évidence leur effet sur les topo-isomérases $[1,2,5]$. La liste des inhibiteurs et leurs mécanismes d'inhibition sont détaillés dans l'article de J.F. Riou et M. Duguet. Les quinolones sont les chefs de file des antibiotiques, tandis que les principaux antitumoraux inhibiteurs de top 2 sont le vépéside (VP-16), la doxorubicine, la mitoxantrone et leurs dérivés, et les acridines et ellipticines. La camptothécine et ses dérivés sont des inhibiteurs de la top 1 . Avec le taxol (paclitaxel) et le taxotère (docitaxel), les inhibiteurs de la top 1 font partie des médicaments les plus prometteurs pour le traitement des tumeurs solides. L'origine naturelle de tous ces produits suggère que la nature a développé et sélectionné ces agents au cours de l'évolution afin de permettre aux organismes de se défendre les uns contre les autres. Il est donc possible qu'existent, dans les cellules, des analogues non toxiques des inhibiteurs intervenant dans la régulation de la réplication et dans les recombinaisons de l'ADN.

La sélectivité des inhibiteurs de la top 2 bactérienne (ou gyrase) s'explique par les différences de séquence, et donc de structure, entre la gyrase et la top 2 humaine [6]. La plus grande sensibilité des cellules malignes aux inhibiteurs de topo-isomérases utilisés en chimiothérapie est moins bien comprise. En effet, leur cible est présente aussi bien dans les cellules normales que dans les cellules tumorales. Certains travaux indiquent que les topo-isomérases sont plus abondantes dans les cellules tumorales. Cette relation directe entre niveau de l'enzyme cible et sensibilité à son inhibiteur peut sembler paradoxale. Elle s'explique par le fait que l'activité cytotoxique des inhibiteurs de topoisomérases n'est pas la conséquence directe de l'inhibition de l'activité enzymatique mais est due au blocage du fonctionnement des barrières topologiques par l'intermédiaire de l'enzyme inhibée. Plus il y a d'enzyme dans la cellule, plus le nombre de barrières bloquées ("points de sabotage ") est élevé et plus la molé- cule est efficace. Le blocage des barrières topologiques n'est pourtant pas suffisant pour expliquer la sélectivité de l'activité cytotoxique des inhibiteurs de topo-isomérases vis-àvis des cellules tumorales. Si l'ADN est au repos, ou si le médicament est éliminé avant réplication ou transcription, les complexes de clivage n'induisent pas de lésions secondaires de l'ADN [5]. La relative incapacité des cellules tumorales à réparer les lésions de l'ADN, leur difficulté à inhiber leur progression dans le cycle cellulaire pour faciliter cette réparation, et leur faculté de s'engager rapidement dans un programme de mort cellulaire par apoptose [7] sont autant de facteurs qui rendent les cellules malignes plus sensibles aux inhibiteurs de topo-isomérases, comme d'ailleurs à d'autres agents tels que les alkylants et, en particulier, le cisplatine.

Plusieurs questions importantes restent à résoudre dans le domaine des topo-isomérases et de leurs inhibiteurs. La première est, sans doute, celle des relations structurales entre les enzymes, l'ADN et les inhibiteurs des enzymes. La réponse passe par la cristallisation de tous les membres de la grande famille des topo-isomérases. La structure cristalline de l'une des deux top 1 bactériennes, la top $\mathrm{A}$, vient d'être identifiée, ainsi que celle de plusieurs régions de la gyrase qui est l'une des deux top 2 bactériennes. Bien que la top A soit très différente de la top 1 humaine (analogue de la top 3 bactérienne) et qu'il n'en existe pas d'homologue dans les cellules eucaryotes, la recherche d'inhibiteurs sera cependant fort intéressante, par exemple en chimiothérapie antimicrobienne. Il reste néanmoins à cristalliser ces enzymes en présence de fragments d'ADN afin de mieux comprendre les mécanismes catalytiques et la structure des intermédiaires enzymatiques formant les barrières topologiques. La cristallisation en présence d'ADN et d'inhibiteurs permettra d'identifier les sites de fixation des différents agents. Son couplage aux méthodes de modélisation moléculaire devrait permettre la création de médicaments ayant des interactions optimales avec les topo-isomérases. 
C'est là une voie prometteuse pour le développement de médicaments plus actifs et plus sélectifs et donc moins toxiques.

Une autre question est de savoir s'il existe d'autres topo-isomérases dans les cellules humaines. Chez les bactéries, deux topo-isomérases de type I (top 1 et top 3) et deux topo-isomérases de type II (gyrase (top 2), et topo IV (top 4 ; parC et parE) [8]) ont été identifiées. Chez l'homme, sont actuellement connues une seule topo-isomérase I (top 1 dont le gène est sur le chromosome 20) et deux topo-isomérases II (top $2 \alpha$ et $\beta$ dont les gènes sont respectivement sur les chromosomes 17 et 3) [6].

L'identification des protéines nucléaires qui règlent l'activité des topo-isomérases et la détermination à l'aide d'inhibiteurs des sites catalytiques dans la chromatine devraient permettre, enfin, de mieux comprendre le rôle des topo-isomérases dans la transcription et les recombinaisons.

Finalement, en dépit de leur efficacité, les inhibiteurs de topo-isomérases donnent lieu à des résistances qui limitent leur activité [5]. Ces résistances sont généralement dues à une diminution d'expression ou à des mutations des topo-isomérases, modifiant des acides aminés de ces enzymes en contact avec les inhibiteurs. Siégeant dans des régions conservées des topo-isomérases, certaines de ces mutations peuvent être identifiées dans des échantillons cliniques. Ces mécanismes de résistance aboutissent à une diminution des complexes de clivage responsables de l'effet thérapeutique des inhibiteurs. Dans l'avenir, l'élucidation des mécanismes de la résistance pourrait conduire à la mise au point de nouveaux produits permettant, seuls ou en association, d'échapper à l'épuisement de l'effet thérapeutique

\section{RÉFÉRENCES}

1. Chen AY, Liu LF. DNA Topoisomerases: essential enzymes and lethal targets. Annu Rev Pharmacol Toxicol 1994 ; 94 : 194218.

2. Pommier Y. DNA topoisomerases I and II in cancer chemotherapy: update and perspectives. Cancer Chemother Pharmacol 1993; 32 : 103-8.

3. Pommier Y, Tanizawa A. Mammalian DNA Topoisomerase I and its inhibitors. In : Hickman J, Tritton T eds. Cancer Chemotherapy. Oxford: Blackwell Scientific Publications LTD, 1993 : 214-50.

4. Lee MP, Brown SD, Hsieh T-S. DNA topoisomerase I is essential in Drosphila Melanogaster. Proc Natl Acad Sci USA 1993 ; $90: 6656-60$.

5. Pommier Y, Leteurtre F, Fesen $M$, Fujimori A, Bertrand R, Solary E, Kohlhagen G, Kohn KW. Cellular determinants of sensitivity and resistance to DNA topoisomerase inhibitors. Cancer Invest $1994 ; 12: 530-42$.

6. Caron PR, Wang JC. DNA Topoisomerases as target of therapeutics : a structural overview. In : Andoh T, Ikeda H, Oguro M eds. Molecular Biology of DNA topoisomerases and its application to chemotherapy. Boca Raton, FL:CRC Press, 1993 : 1-18.

7. Solary E, Bertrand R, Pommier Y. Le rôle de l'apoptose dans la genèse et le traitement du cancer. médecine/sciences $1993 ; 9: 667-75$.

8. Kato JI, Suzuki H, Ikeda H. Purification and characterization of DNA topoisomerase IV in Escherichia coli. J Biol Chem 1992 ; 267 : 25676-84.

\section{Remerciements}

Nous remercions le Dr Éric Solary et le Dr Yves Champey pour leurs critiques et suggestions lors de la rédaction de cet article.

\section{TIRÉS A PART}

Y. Pommier. 